\title{
Comparative study on first and second order ILC - frequency domain analysis and experiments
}

\author{
Mikael Norrlöf \\ Division of Automatic Control \\ Department of Electrical Engineering \\ Linköpings universitet, SE-581 83 Linköping, Sweden \\ WWW: http://www. control.isy.liu.se \\ Email: mino@isy.liu.se
}

17th August 2001

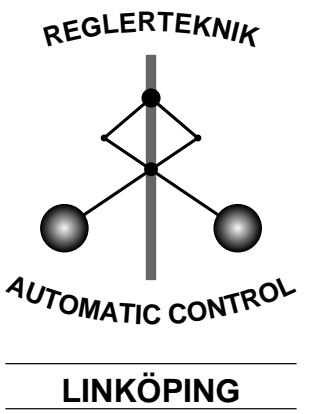

Report No.: LiTH-ISY-R-2373

Submitted to 2000 IEEE Conference on Decision and Control, Sydney, Australia

Technical reports from the Automatic Control group in Linköping are available by anonymous ftp at the address ftp.control.isy.liu.se. This report is contained in the file 2373.pdf. 


\begin{abstract}
Aspects on the behavior of a general second order iterative learning control (ILC) algorithm are presented from a frequency domain perspective. This includes stability as well as performance and robustness issues. The basis for the analysis is linear iterative systems and these are briefly described. A design algorithm for second order ILC schemes is proposed and analyzed both theoretically as well as in an experiment. In the experiment, done on a commercial industrial robot control system, the second order ILC design is compared with a first order ILC design. The result from both the analysis and the experiment is that the second order design is not better with respect to performance or robustness.
\end{abstract}

Keywords: iterative learning control, ilc, high order ilc, experiment, robot 


\title{
Comparative study on first and second order ILC - frequency domain analysis and experiments*
}

\author{
M. Norrlöf \\ Department of Electrical Engineering, Linköpings universitet, \\ SE-581 83 Linköping, Sweden \\ Email: mino@isy.liu.se
}

\begin{abstract}
Aspects on the behavior of a general second order iterative learning control (ILC) algorithm are presented from a frequency domain perspective. This includes stability as well as performance and robustness issues. The basis for the analysis is linear iterative systems and these are briefly described. A design algorithm for second order ILC schemes is proposed and analyzed both theoretically as well as in an experiment. In the experiment, done on a commercial industrial robot control system, the second order ILC design is compared with a first order ILC design. The result from both the analysis and the experiment is that the second order design is not better with respect to performance or robustness.
\end{abstract}

\section{Introduction}

It is a fact that many systems in applications like robotics repeat the same actions over and over again. Often it is also the case that there is a difference between what should be achieved and what is actually achieved. The idea of iterative learning control (ILC) is to use the information from previous iterations in such a way that the difference between what should be achieved and what actually is achieved eventually becomes smaller. The first papers on this topic are from 1984, [1, 2, 3], and since then a lot of publications have been published. Just to mention a few $[4,5,6]$ that can serve as a deeper introduction to the topic.

The contributions in this paper are: An analysis of the behavior of second order ILC systems from a transient and asymptotic point of view, and a proposed design scheme, which is also tested on an industrial robot control system. The result from using the second order ILC algorithm is also compared with that of a first order ILC design. Second

\footnotetext{
* This work was supported by ABB Robotics within NUTEK's Center of Excellence ISIS at Linköpings universitet.
}

and higher order ILC methods have been analyzed in previous works, e.g., [7, 8, 6] and [9]. What has not been so much addressed, however, is what kind of transient behavior can be expected and how the filters in the second order ILC scheme shall be chosen.

\section{Problem formulation}

The discussion will be restricted to linear time invariant SISO systems that can be described in discrete time by

$$
y_{k}(t)=T_{r}(q) r(t)+T_{u}(q) u_{k}(t)
$$

where $y_{k}(t)$ is the output, $r(t)$ is the desired output, $u_{k}(t)$ is the control signal that can be modified by the ILC algorithm, and $k$ is the iteration number. The signals are all assumed to be defined on a time interval $t \in\left[0, t_{f}\right]$. The transfer operators $T_{r}(q)$ and $T_{u}(q)$ are assumed to be stable discrete time filters.

The system formulation in (1) is discussed in more detail in [10] and it covers easily both open loop as well as closed loop systems. It should be stressed however that here it will be assumed that a feedback control solution is applied before introducing ILC. This means that ILC will be considered as being a control strategy applied in addition to feedback control, not instead of feedback control.

In this paper a first and a second order ILC algorithm will be considered. By defining the error, $e_{k}(t) \triangleq r(t)-y_{k}(t)$, the first order ILC updating equation becomes,

$$
u_{k+1}(t)=Q(q)\left(u_{k}(t)+L(q) e_{k}(t)\right)
$$

while the second order becomes,

$$
\begin{aligned}
u_{k+1}(t)= & Q_{1}(q)\left(u_{k}(t)+L_{1}(q) e_{k}(t)\right) \\
& +Q_{2}(q)\left(u_{k-1}(t)+L_{2}(q) e_{k-1}(t)\right)
\end{aligned}
$$

For the analysis of the second order ILC algorithm the notion of linear iterative systems will be used. These are presented briefly in the next section. 


\section{Linear Iterative Systems}

A linear iterative system can be described as

$$
z_{k+1}(t)=F(q) z_{k}(t)+F_{r}(q) r(t)
$$

where $z_{k}(t) \in \mathbb{R}^{N}, r(t) \in \mathbb{R}$, and $F(q), F_{r}(q)$ are matrices of discrete time transfer operators. In addition it is assumed that $t$ is limited, $0 \leq t \leq t_{f}$. In the analysis of high order ILC systems, $z_{k}(t)$ can be chosen as $\left[u_{k}(t), u_{k-1}(t) \ldots u_{k-N+1}(t)\right]^{T}$. See [10] for more details. Now, a theorem that gives the condition for bounded input bounded output (BIBO) stability of a linear iterative systems is presented.

Theorem 1 (BIBO stability) The iterative system given by (4) is BIBO stable if

$$
\bar{\rho}=\sup _{\omega \in\left[0, \pi / t_{s}\right]} \rho\left(F\left(e^{i \omega}\right)\right)<1
$$

where $\rho(\cdot)$ is the spectral radius of the matrix $F\left(e^{i \omega}\right)$.

For the proof see [10]. $F\left(e^{i \omega}\right)$ is the frequency domain representation of $F(q)$. The intuitive interpretation of this theorem is that, if we have a vector of a certain size (norm) and this vector eventually is mapped into a vector of smaller size (norm), then we have a stable iterative mapping function. Having the spectral radius smaller than one guarantees that the size eventually will become smaller. When the linear iterative system in (4) is BIBO stable, the asymptotic value of $z_{k}(t)$ can be calculated as

$$
\lim _{k \rightarrow \infty} z_{k}(t)=z_{\infty}(t)=(I-F(q))^{-1} F_{r}(q) r(t)
$$

The fact that higher order systems can be written in this form has also been explored in, e.g., [11] and [12].

\section{Stability analysis}

The results from the previous section can be used for the analysis of ILC systems. Here only first order and second order ILC algorithms will be discussed but it is straightforward to extend the results to higher order ILC systems.

\subsection{First order ILC}

Using the first order ILC updating formula given by (2) on the system described by (1) it results in an updating equation for $u_{k}$ according to

$$
\begin{aligned}
u_{k+1}(t)= & Q(q)\left(\left(1-L(q) T_{u}(q)\right) u_{k}(t)\right. \\
& \left.+L(q)\left(1-T_{r}(q)\right) r(t)\right)
\end{aligned}
$$

Now, let $F(q)=Q(q)\left(1-L(q) T_{u}(q)\right)$ and $F_{r}(q)=$ $L(q)\left(1-T_{r}(q)\right)$. This means that it is possible to write (6) in the same form as (4) and from Theorem 1 the well known stability criterion follows,

$$
\left|1-L\left(e^{i \omega}\right) T_{u}\left(e^{i \omega}\right)\right|<\left|Q^{-1}\left(e^{i \omega}\right)\right|, \quad \forall \omega
$$

where the $Q$-filter can be used to increase the stability region. Notice however that by choosing a $Q$ different from 1 the asymptotic error will no longer be guaranteed to be zero.

\subsection{Second order ILC}

When using the second order ILC updating formula (3) on the system described by (1) the resulting update equation becomes,

$$
\begin{aligned}
u_{k+1}(t) & =Q_{1}(q)\left(\left(1-L_{1}(q) T_{u}(q)\right) u_{k}(t)\right) \\
& +Q_{2}(q)\left(1-L_{2}(q) T_{u}(q)\right) u_{k-1}(t) \\
& +\left(Q_{1}(q) L_{1}(q)+Q_{2}(q) L_{2}(q)\right)\left(1-T_{r}(q)\right) r(t)
\end{aligned}
$$

Let

$$
\begin{aligned}
& z_{k}(t)=\left[\begin{array}{c}
u_{k}(t) \\
u_{k-1}(t)
\end{array}\right], F(q)=\left[\begin{array}{cc}
F_{1}(q) & F_{2}(q) \\
1 & 0
\end{array}\right] \\
& F_{r}(q)=\left[\begin{array}{c}
\left(Q_{1}(q) L_{1}(q)+Q_{2}(q) L_{2}(q)\right)\left(1-T_{r}(q)\right) \\
0
\end{array}\right]
\end{aligned}
$$

with $F_{1}(q)=Q_{1}(q)\left(1-L_{1}(q) T_{u}(q)\right)$ and $F_{2}(q)=$ $Q_{2}(q)\left(1-L_{2}(q) T_{u}(q)\right)$ it is possible to apply the general stability result of Theorem 1 . To guarantee that the asymptotic error decreases to zero $Q_{1}+Q_{2}$ has to be chosen equal to one, see [10] for the proof. Compare this with the condition $Q \equiv 1$ for guaranteed zero error convergence in the first order case in Section 4.1.

\section{Convergence behavior}

It is not only the stability of the ILC system that is important. Also the performance should be considered. The convergence speed is here analyzed from a frequency domain perspective. For a more detailed discussion the reader is referred to [13] and [10].

\subsection{First order ILC}

When considering convergence speed it is important to see how fast the system with the ILC algorithm converges to a pre-specified trajectory. This is the same as studying how

$$
\tilde{U}_{k, \omega}^{1}\left(e^{i \omega}\right)=U_{\infty}\left(e^{i \omega}\right)-U_{k}\left(e^{i \omega}\right)
$$


converges to zero. Using the first order ILC scheme in (2), the definition of the error $E_{k}\left(e^{i \omega}\right)=R\left(e^{i \omega}\right)-Y_{k}\left(e^{i \omega}\right)$, and the system description in (1) it follows that

$$
\tilde{U}_{k+1, \omega}^{1}\left(e^{i \omega}\right)=Q\left(e^{i \omega}\right)\left(1-L\left(e^{i \omega}\right) T_{u}\left(e^{i \omega}\right)\right) \tilde{U}_{k, \omega}^{1}\left(e^{i \omega}\right)
$$

With $F\left(e^{i \omega}\right)=Q\left(e^{i \omega}\right)\left(1-L\left(e^{i \omega}\right) T_{u}\left(e^{i \omega}\right)\right)$ this means that

$$
\left|\tilde{U}_{k, \omega}\right|=\left|F\left(e^{i \omega}\right)\right|^{k}\left|\tilde{U}_{0, \omega}\right|
$$

which gives a clear message about the convergence. It is exponential with the rate decided by the frequency function $\left|F\left(e^{i \omega}\right)\right|$.

\subsection{Second order ILC}

First the main result for second order ILC systems is presented.

Theorem 2 (Eigenvalue decomposition) It is possible to write $\tilde{U}_{k, \omega}$ on the following decomposed form,

$$
\tilde{U}_{k}\left(e^{i \omega}\right)=\tilde{U}_{0}\left(e^{i \omega}\right)\left(\kappa_{1, \omega} \lambda_{1, \omega}^{k+1}+\kappa_{2, \omega} \lambda_{2, \omega}^{k+1}\right)
$$

where $\tilde{U}_{k}\left(e^{i \omega}\right)$ is defined according to (9) and,

$$
\kappa_{1, \omega}=\frac{1-\lambda_{2, \omega}}{\lambda_{1, \omega}-\lambda_{2, \omega}}, \quad \kappa_{2, \omega}=\frac{\lambda_{1, \omega}-1}{\lambda_{1, \omega}-\lambda_{2, \omega}}
$$

and $\lambda_{1, \omega}, \lambda_{2, \omega}$ are the eigenvalues of the matrix $F\left(e^{i \omega}\right)$ from (8).

The proof (see [10]) is based on the fact that $\tilde{Z}_{k}\left(e^{i \omega}\right)=$ $Z_{\infty}\left(e^{i \omega}\right)-Z_{k}\left(e^{i \omega}\right)$ can be written in the base constructed from the eigenvectors of the matrix $F\left(e^{i \omega}\right)$ at every frequency. The result in Theorem 2 is the basis for the results on the behavior of $\left|\tilde{U}_{k, \omega}\right|$.

\section{Design}

Before going into the actual design example and the experiments, some general comments on the choices of eigenvalues that are actually reasonable.

The eigenvalues of the matrix in (8) can be calculated using

$$
\lambda_{(1,2), \omega}=\frac{F_{1}\left(e^{i \omega}\right)}{2} \pm \sqrt{\frac{\left(F_{1}\left(e^{i \omega}\right)\right)^{2}}{4}+F_{2}\left(e^{i \omega}\right)}
$$

with $F_{1}\left(e^{i \omega}\right)$ and $F_{2}\left(e^{i \omega}\right)$ as the frequency domain representations of the corresponding $F_{1}(q)$ and $F_{2}(q)$ in (8).

From now on it is assumed that the system where ILC is applied can be described as in (1). The proposed second order ILC updating formula is given by (3). A design methodology for this ILC algorithm has to be able to find the filters $Q_{1}, Q_{2}, L_{1}$, and $L_{2}$. How to choose the $Q$ and the $L$ filters in a first order ILC formulation is quite well known and there exist some algorithms that from a model can compute the filters, see for example $[6,4,10]$.

\subsection{Design algorithm proposal}

By considering (11) it is clear that given a value of $F_{1}\left(e^{i \omega}\right)$ the best eigenvalue, with respect to the amplitude, is $\frac{F_{1}\left(e^{i \omega}\right)}{2}$. This is the case when $F_{2}\left(e^{i \omega}\right)$ is chosen such that

$$
\frac{\left(F_{1}\left(e^{i \omega}\right)\right)^{2}}{4}+F_{2}\left(e^{i \omega}\right)=0
$$

which is equivalent to

$$
\begin{aligned}
& Q_{2}\left(e^{i \omega}\right)=-\frac{\left(Q_{1}\left(e^{i \omega}\right)\right)^{2}}{4} \\
& L_{2}\left(e^{i \omega}\right)=L_{1}\left(e^{i \omega}\right)\left(2-L_{1}\left(e^{i \omega}\right) T_{u}\left(e^{i \omega}\right)\right)
\end{aligned}
$$

This choice will, however, only fulfill the condition $Q_{1}+$ $Q_{2} \equiv 1$, mentioned in Section 4.2, if $Q_{1}=2$. The approach here will instead be to choose an approximate solution based on a first order ILC design.

\section{Algorithm 1 (Second order ILC design)}

1. Design a first order ILC algorithm, i.e., choose the filters $Q$ and $L$ according to one design methodology for first order ILC algorithms.

2. Choose $Q_{1}$ and $L_{1}$ according to,

$$
Q_{1}\left(e^{i \omega t_{s}}\right)=\frac{5}{4} Q\left(e^{i \omega}\right), \quad L_{1}\left(e^{i \omega}\right)=L\left(e^{i \omega}\right)
$$

3. Choose $Q_{2}$ and $L_{2}$ such that

$$
\begin{aligned}
& Q_{2}\left(e^{i \omega}\right)=-\frac{\left(Q\left(e^{i \omega}\right)\right)^{2}}{4} \\
& L_{2}\left(e^{i \omega}\right)=L_{1}\left(e^{i \omega}\right)\left(2-L_{1}\left(e^{i \omega}\right) T_{u}\left(e^{i \omega}\right)\right)
\end{aligned}
$$

The approach suggested in Algorithm 1 is model based since $T_{u}$ is used in the construction of the $L_{2}$ filter. One important difference compared to many suggested first order ILC design schemes is however that it is the model and not its inverse that is included. If the first order ILC algorithm is chosen as the optimal solution $L_{1}=T_{u}^{-1}$, without considering robustness, then from the choice of $L_{2}$ in the algorithm it is obvious that also $L_{2}=T_{u}^{-1}$. The choice of $Q_{1}$ and $Q_{2}$ stem from the condition mentioned in Section 4.2. In the frequency band where the first order ILC system has zero error convergence, i.e., $Q_{\omega}=1$, the second order design using Algorithm 1 will also converge to zero since

$$
Q_{1}\left(e^{i \omega}\right)+Q_{2}\left(e^{i \omega}\right)=\frac{5}{4} Q\left(e^{i \omega}\right)-\frac{\left(Q\left(e^{i \omega}\right)\right)^{2}}{4} \approx 1
$$




\subsection{Analysis of resulting design}

A natural way of evaluating the second order ILC design is to compare it with the corresponding first order design, both from a performance as well as a robustness point of view.

\subsubsection{Performance and robustness for a first order ILC}

From Section 5.1 it is clear that the first order ILC algorithm will give an exponential convergence in the frequency domain. The rate will depend on the frequency function $F\left(e^{i \omega}\right)=Q\left(e^{i \omega}\right)\left(1-L\left(e^{i \omega}\right) T_{u}\left(e^{i \omega}\right)\right)$.

Assume that there is a relative model uncertainty $\Delta_{r}\left(e^{i \omega}\right)$

$$
F\left(e^{i \omega}\right)=\bar{F}\left(e^{i \omega}\right)\left(1+\Delta_{r}\left(e^{i \omega}\right)\right), \quad\left|\Delta_{r}\left(e^{i \omega}\right)\right|<\gamma(\omega)
$$

where $\bar{F}\left(e^{i \omega}\right)$ is the nominal value. A sufficient condition for stability becomes

$$
\gamma(\omega)<\frac{1}{\left|\bar{F}\left(e^{i \omega}\right)\right|}-1
$$

If there is an absolute uncertainty,

$$
F\left(e^{i \omega}\right)=\bar{F}\left(e^{i \omega}\right)+\Delta_{a}\left(e^{i \omega}\right), \quad\left|\Delta_{a}\left(e^{i \omega}\right)\right|<\gamma(\omega)
$$

the corresponding sufficient criterion for robust stability becomes

$$
\gamma(\omega)<1-\left|F\left(e^{i \omega}\right)\right|
$$

Now the proposed second order ILC design can be compared with the first order ILC scheme.

\subsubsection{Eigenvalue based design}

When using Algorithm 1 the eigenvalues are given by

$$
\lambda_{(1,2), \omega}=\frac{Q\left(e^{i \omega}\right)\left(1-L\left(e^{i \omega}\right) T_{u}\left(e^{i \omega}\right)\right)}{8}(5 \pm 3)
$$

Obviously this means that if $F\left(e^{i \omega}\right)=Q\left(e^{i \omega}\right)(1-$ $\left.L\left(e^{i \omega}\right) T_{u}\left(e^{i \omega}\right)\right)$ is the result of the original first order ILC design, the nominal eigenvalues are $\lambda_{1, \omega}=F\left(e^{i \omega}\right)$ and $\lambda_{2, \omega}=\frac{1}{4} F\left(e^{i \omega}\right)$. From a robustness perspective this is exactly the same result as for the first order ILC algorithm since the amplitude margin for the second order case,

$$
1-\max \left(\left|\lambda_{1, \omega}\right|,\left|\lambda_{2, \omega}\right|\right)=1-\left|F\left(e^{i \omega}\right)\right|
$$

is exactly the same as the one of the first order.

It is also important to consider the performance that can be achieved compared to the first order ILC design. For the second order design it follows from Theorem 2 that

$$
\frac{\left|\tilde{U}_{k, \omega}^{2}\right|}{\left|\tilde{U}_{0, \omega}^{2}\right|}=\left|F\left(e^{i \omega}\right)\right|^{k} \frac{1}{3 \cdot 4^{k}}\left|4^{k+1}-1-F\left(e^{i \omega}\right)\left(4^{k}-1\right)\right|
$$

To compare the first and the second order ILC algorithms consider,

$$
\frac{\left|\tilde{U}_{k, \omega}^{2}\right|}{\left|\tilde{U}_{0, \omega}^{2}\right|}-\frac{\left|\tilde{U}_{k, \omega}^{1}\right|}{\left|\tilde{U}_{0, \omega}^{1}\right|}
$$

Using the following bound

$$
1<\frac{1}{3 \cdot 4^{k}}\left|4^{k+1}-1-F\left(e^{i \omega}\right)\left(4^{k}-1\right)\right|<\frac{5}{3}
$$

which is found using the triangular inequality and the fact that $\left|F\left(e^{i \omega}\right)\right|<1$. It is obvious that the second order ILC algorithm designed using the eigenvalue based design will never work better compared to the first order ILC design on which it is based.

\section{Experiment}

The theory developed in the previous sections can now be applied on a design example for a real industrial system. The system, an ABB IRB1400 industrial robot, is depicted in Figure 1. For a more thorough description of the technical part of the experimental setup see [10].

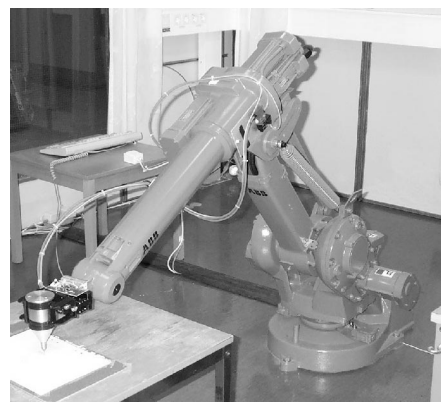

Figure 1. The ABB IRB1400 manipulator.

In this example ILC is applied to three of the robot's six joints. Each of the three joints is modeled as a transfer operator description from the ILC control input to the measured motor position on the robot, i.e., $T_{u}$ in (1). It should be stressed that $T_{u}$ is in fact a model of a closed loop system. The conventional feedback controller in the S4C control system is working in parallel with the ILC scheme. The models, $T_{u}$ are calculated using System Identification Toolbox [14] and are given by,

$$
\begin{aligned}
\hat{T}_{u_{1}}(q)= & \hat{T}_{u_{2}}(q)=\frac{0.1 q^{-1}}{1-0.9 q^{-1}} \\
\hat{T}_{u_{3}}(q) & =\frac{0.13 q^{-1}}{1-0.87 q^{-1}}
\end{aligned}
$$



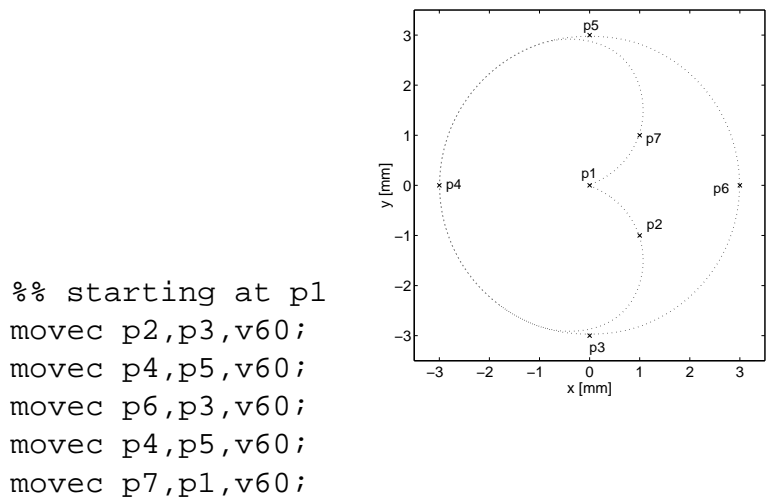

Figure 2. The program that produces the trajectory used in the example (left) and the resulting trajectory translated such that the origin coincide with p1 (right).

\subsection{Description of the experiment}

The experiment is done on the ABB IRB1400 robot in the research lab of Division of Automatic Control at Linköping University. In Figure 2 the program used in the experiment is shown together with the resulting trajectory on the armside of the robot. The instruction movec p2, p3, v60 refers to an instruction that produces an arc on the arm-side of the robot. The arc starts from the current position, not explicitly stated, and goes through the points $\mathrm{p} 2$ and $\mathrm{p} 3$. The speed along the path is programmed to be $60 \mathrm{~mm} / \mathrm{s}$. Actual position of $\mathrm{p} 1$ in the base coordinate system is $\mathrm{x}=1300$ $\mathrm{mm}, \mathrm{y}=100 \mathrm{~mm}$, and $\mathrm{z}=707 \mathrm{~mm}$. The configuration of the robot is also shown in Figure 1.

\subsection{ILC design}

The design of the first order ILC scheme is based on an algorithm discussed in, e.g., [10]. The procedure will only be briefly described here.

The filter $L$ is chosen as

$$
L(q)=0.9 q^{2}
$$

The corresponding $Q$ filter is chosen as $Q\left(e^{i \omega}\right)=$ $Q_{1 / 2}\left(e^{i \omega}\right) Q_{1 / 2}\left(e^{-i \omega}\right)$, i.e., a zero-phase filter, with $Q_{1 / 2}(q)$ as a second order Butterworth filter with cut-off frequency 0.2 of the Nyquist frequency. The resulting nominal $Q\left(e^{i \omega}\right)\left(1-L\left(e^{i \omega}\right) T_{u_{i}}\left(e^{i \omega}\right)\right)$ are depicted in Figure 3 and it is obvious that the convergence criterion in (7), is fulfilled for all the three joints.

The second order ILC algorithm is designed based on the first order ILC design according to Algorithm 1.
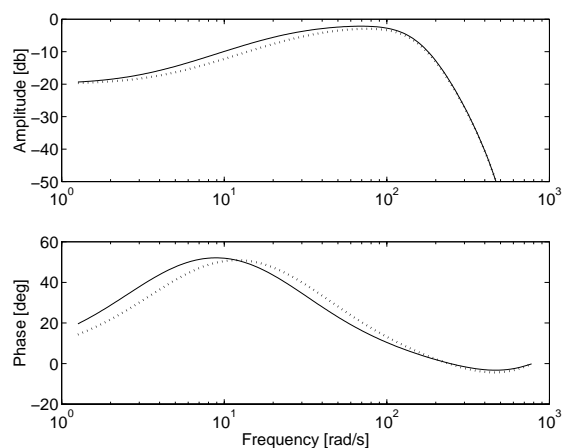

Figure 3. The results from the design of the first order ILC algorithm. $Q\left(e^{i \omega}\right)(1-$ $\left.L\left(e^{i \omega}\right) T_{u_{i}}\left(e^{i \omega}\right)\right)$, for $i=1,2$ (solid line) and $i=3$ (dotted).

\subsection{Results from the experiments}

The result from the experiments can be evaluated from two different points of view. First the result achieved on the motor side can be studied. This is the measure used by the ILC algorithms and it is the error in this measure that will be minimized. Clearly, from an application's point of view it is more interesting to minimize the arm side error. The industrial robot does not have this possibility today since the only measurements that are available come from the motors. The motors are then connected to the arms through gear-boxes which in them self are dynamic systems. Using models it is possible to predict how the arm will move and generate a trajectory for the motors that will produce the desired path on the arm-side. We will assume that the given motor trajectory is correct with respect to the gear-box and arm dynamics.

\subsubsection{Motor-side}

The two ILC algorithms have run for 10 iterations. Since in the first iteration ILC is not applied, $u_{0} \equiv 0$, the same circle has been done 11 times. In Figure 4 the resulting normalized $\infty$-norm and 2-norm of the error on the motor-side are shown. The $\infty$-norm is simply defined as the maximum value of the absolute value of the error. The 2-norm is instead a measure of the root mean square of the error.

In the first iteration both the algorithms use the same updating equations and should,in theory, therefore also reach the same level of error. As can be seen in Figure 4 this is not the case. The ILC designed according to Algorithm 1 gives a lower value of the error in the first iteration. In the second iteration the size of the error is again about the same and after the fifth iteration the error stabilizes on a level where 

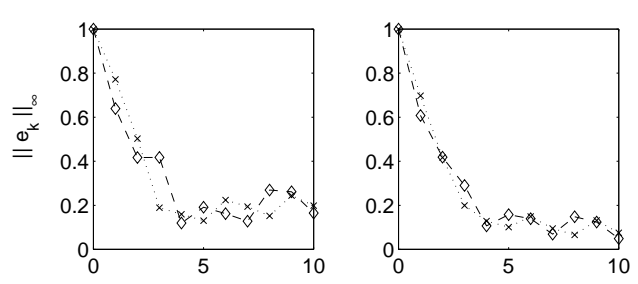

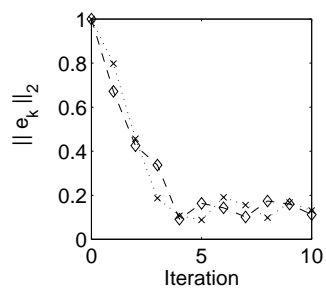

(a) Motor 1 .

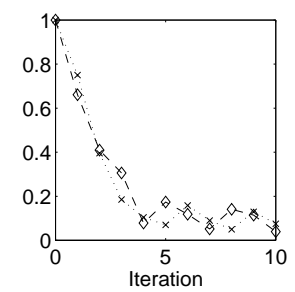

(b) Motor 2 .
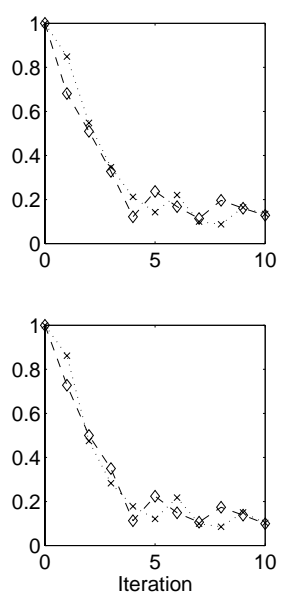

(c) Motor 3 .

Figure 4. The errors in $\infty$-norm and 2-norm for the two algorithms. First order ILC design $(\times)$ and Algorithm $1(\diamond)$.

$\left\|e_{k}\right\|_{\infty}$ is about $15 \%$ of the initial value. The reason why the error does not converge to zero is that there are disturbances acting on the system in the form of measurement disturbances and load disturbances. The load disturbance comes from the fact that the robot actually is in contact with the surface of the table when it draws the circles. The measurement disturbances also gives a remaining error which will not be possible to remove by using the proposed ILC schemes since they are, in general, not periodic with respect to the iterations.

\subsubsection{Arm-side}

When evaluating the result of the ILC iterations on the armside of the robot a pen is used, as shown in Figure 1. The resulting drawings are not so easy to evaluate since the pen in itself produces a quite thick line. In Figure 5 the resulting circles are shown for the two different approaches for the first 5 iterations (iteration 0 to 5 , from left to right). Clearly the error reduces also on the arm-side as it was on the motorside. In an application, such as laser cutting, the accuracy demand is an absolute error of about $0.1 \mathrm{~mm}$. Using this setup with the pen mounted on the robot is not sufficient to evaluate this accuracy.

It is also possible to evaluate the result from the ILC experiments on the arm-side by doing a transformation of the measured motor angles to the arm side using the forward kinematic model of the IRB1400, see e.g., [15]. The forward kinematic model maps the motor angles into a tool position and orientation, although only the position has been used here. This transformation relies on the fact that the robot is totally rigid which works well in steady state but not as well when the robot is moving. It does, however, give an idea on how well an idealized robot would follow the desired path and it also tells us how well the real robot is approximated by this idealized case if we compare the result in Figure 6 and the result in Figure 5. Clearly a similar behavior is achieved in the two cases. Notice that although the error is reduced monotonically on the motor-side this does not have to be the case on the arm-side. The error is actually bigger on the arm-side in iterations 1 and 2 compared to iteration 0 although, on the motor-side, the error is reduced in every iteration evaluated in both norms.

\section{Conclusion}

The analysis of second order ILC systems based on linear iterative systems is very promising and gives a lot of insight into the behavior of second order ILC schemes. From the results presented in the paper it is not possible to say that a second order ILC algorithm does better than a first order algorithm. From the analysis and, in fact, also the experiments it is evident that it works as well as the first order design. Some facts are however important to stress when thinking of moving from a first order ILC design to a second order ILC design.

- The second order design should not use more information about the system than the first order design.

- The amount of memory required for the second order ILC scheme, as implemented in this paper, is double the amount used by the corresponding first order ILC scheme. 
- One aspect that has not been considered in this paper but that can make the second order ILC scheme very competitive is when there is an uncertainty in the plant that makes the plant different between iterations. The second order algorithm can smooth the behavior of the system by using the control and the error signal from more than one iteration.

Further work in the area could be to consider the effects of nonlinearities on the resulting control signals and the resulting error. For example, Coloumb friction is a nonlinear effect that is always present in real servo systems and a comparison between a first and second order ILC algorithms for dealing with this could be worth to consider.

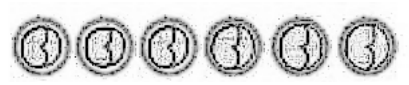

(a) First order ILC.

\section{(2)(1) (a) (1) (2) (2)}

(b) Second order ILC. Figure 5. Result on arm-side of the ILC itera-
tions 0 to 5 (from left to right).

\section{References}

[1] S. Arimoto, S. Kawamura, and F. Miyazaki. Bettering operation of robots by learning. Journal of Robotic Systems, 1(2):123-140, 1984.

[2] G. Casalino and G. Bartolini. A learning procedure for the control of movements of robotic manipulators. In IASTED Symposium on Robotics and Automation, pages 108-111, 1984.

[3] J.J. Craig. Adaptive control of manipulators through repeated trials. In Proc. of ACC, San Diego, CA, June 1984.

[4] K. L. Moore. Iterative Learning Control for Deterministic Systems. Advances in Industrial Control. Springer-Verlag, 1993.

[5] K. L. Moore. Iterative learning control - an expository overview. Applied and Computational Controls, Signal Processing and Circuits, 1, 1998.

[6] Z. Bien and J.-X. Xu. Iterative Learning Control: Analysis, Design, Integration and Application. Kluwer Academic Publishers, 1998.
[7] Z. Bien and K.M. Huh. Higher-order iterative learning control algorithm. In IEE Proceedings, volume 136, pages 105 - 112, May 1989.

[8] Y. Chen, Z. Gong, and C. Wen. Analysis of a high order iterative learning control algorithm for uncertain nonlinear systems. Automatica, 34(3):345-353, March 1998.

[9] M. Norrlöf and S. Gunnarsson. A frequency domain analysis of a second order iterative learning control algorithm. In Proc. of the 38th IEEE Conference on Decision and Control, Pheonix, Arizona, USA, Dec 1999.

[10] M. Norrlöf. Iterative Learning Control: Analysis, Design, and Experiments. $\mathrm{PhD}$ thesis, Linköping University, Linköping, Sweden, 2000. Linköping Studies in Science and Technology. Dissertations No. 653.

[11] E. Rogers and D. H Owens. Stability Analysis for Linear Repetitive Processes, volume 175 of Lecture Notes in Control and Information Sciences. Springer-Verlag, 1992.

[12] N. Amann, D. H. Owens, and E. Rogers. 2d systems theory applied to learning control systems. In Proc. of the 33rd IEEE Conf. on Decision and Control, Lake Buena Vista, FL, USA, Dec 1994.

[13] M. Norrlöf. Analysis of a second order iterative learning controller. Technical Report LiTH-ISY-R-2181, Department of Electrical Engineering, Linköping University, Feb 2000.

[14] L. Ljung. System Identification Toolbox - For Use with Matlab. The MathWorks Inc., 1995.

[15] M. Norrlöf. Modeling of industrial robots. Technical Report LiTH-ISY-R-2208, Department of Electrical Engineering, Linköping University, Dec 1999. 

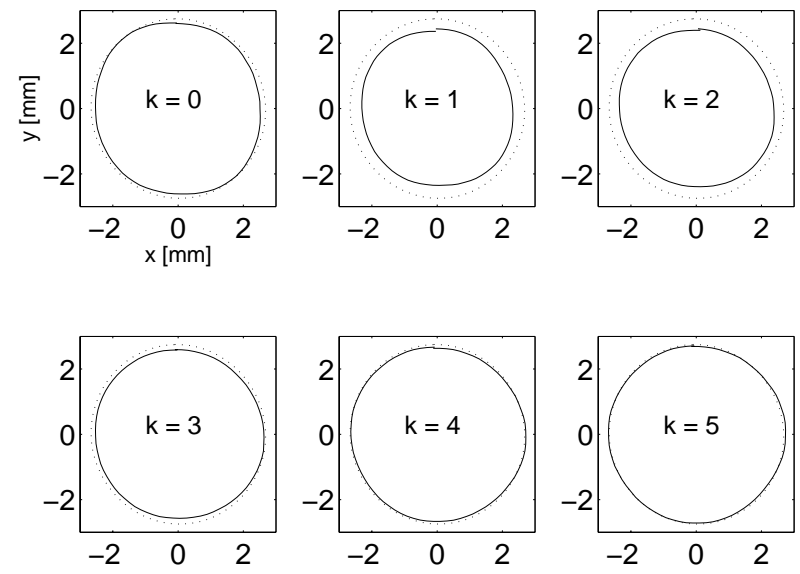

(a) First order ILC.
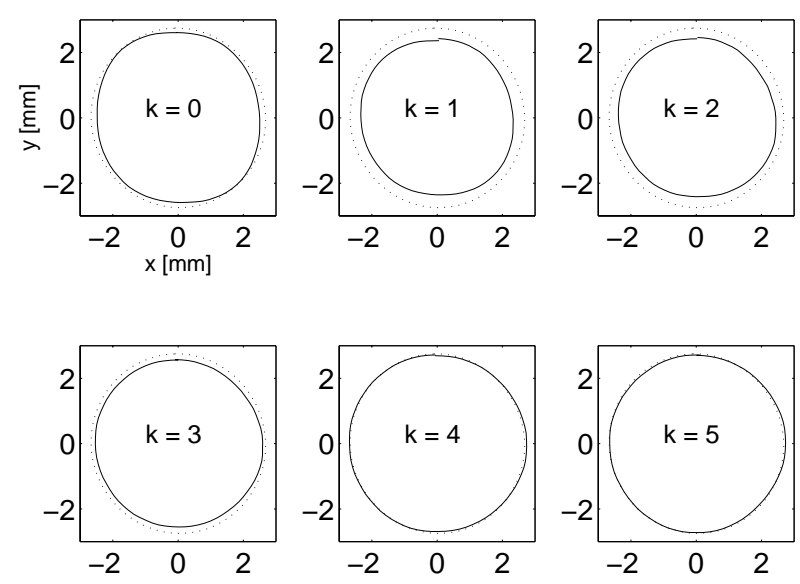

(b) Second order ILC.

Figure 6. Results from kinematic transformation from motor to arm. 